\title{
Landslide susceptibility mapping at VAZ watershed (Iran) using an artificial neural network model: a comparison between multilayer perceptron (MLP) and radial basic function (RBF) algorithms
}

\begin{abstract}
Landslide susceptibility and hazard assessments are the most important steps in landslide risk mapping. The main objective of this study was to investigate and compare the results of two artificial neural network (ANN) algorithms, i.e., multilayer perceptron (MLP) and radial basic function (RBF) for spatial prediction of landslide susceptibility in Vaz Watershed, Iran. At first, landslide locations were identified by aerial photographs and field surveys, and a total of 136 landside locations were constructed from various sources. Then the landslide inventory map was randomly split into a training dataset $70 \%$ (95 landslide locations) for training the ANN model and the remaining $30 \%$ (41 landslides locations) was used for validation purpose. Nine landslide conditioning factors such as slope, slope aspect, altitude, land use, lithology, distance from rivers, distance from roads, distance from faults, and rainfall were constructed in geographical information system. In this study, both MLP and RBF algorithms were used in artificial neural network model. The results showed that MLP with Broyden-Fletcher-Goldfarb-Shanno learning algorithm is more efficient than RBF in landslide susceptibility mapping for the study area. Finally the landslide susceptibility maps were validated using the validation data (i.e., $30 \%$ landslide location data that was not used during the model construction) using area under the curve (AUC) method. The success rate curve showed that the area under the curve for RBF and MLP was $0.9085(90.85 \%)$ and $0.9193(91.93 \%)$ accuracy, respectively. Similarly, the validation result showed that the area under the curve for MLP and RBF models were 0.881 (88.1 \%) and 0.8724 (87.24 \%), respectively. The results of this study showed that landslide susceptibility mapping in the Vaz Watershed of Iran using the ANN approach is viable and can be used for land use planning.
\end{abstract}

Keyword: Landslide, Susceptibility, Artificial neural networks, Geographic Information Systems (GIS), Vaz Watershed, Iran 